\title{
Contribution à l'étude des Cératopogonidés de Suisse. III. Description de trois espèces nouvelles appartenant au genre Forcipomyia Meigen (Diptera, Nematocera)
}

\author{
par Jean-Claude DELÉCOLLE * et Karin SChiEGG ** \\ * Musée Zoologique de l'Université Louis Pasteur et de la Ville de Strasbourg (MZS). 29 boulevard de la \\ Victoire, F-67000 Strasbourg. ${ }^{* *}$ Chaire de la protcction de la nature et du paysage, Instilul fédéral de \\ recherches sur la forêt, la neige et le paysage, 111 Zürcherstrasse, $\mathrm{CH}-8903$ Birmensdorf, Suisse
}

Résumé. - Forcipomyia pseudonigra, $F$. sihlwaldensis et $F$. helvetica, nouvelles espèces de Cératopogonidés originaires de Suisse, sont décrites et illustrées.

Summary. - Contribution to the study of the Ceratopogonidae from Switzerland. III. Description of three new species of the genus Forcipomyia Meigen (Diptera, Nematocera). Forcipomyia pseudonigra, $F$. sihlwaldensis and $F$. helvetica, new species of biting midge from Switzerland, are described and illustrated.

Mots clés. - Diptera, Ceratopogonidae, Forcipomyia pseudonigra, F. sihlwaldensis, F. helvetica, n. spp., Suisse.

Poursuivant l'étude des Cératopogonidés capturés en 1996 et 1997, à l'aide d'un piège attractif "Trunk Window Trap" ou TWT (KAILA, 1993) et d'un piège à émergences "Baumstammeklektor" ou EK (FUNKE, 1971), dans la forêt du Sihlwald, près de Zurich $\left(47^{\circ} 15^{\prime} \mathrm{N}, 8^{\circ} 33^{\prime} \mathrm{E}\right)$, et dont une partie a déjà fait l'objet de deux publications (DELÉCOLLL \& SCHIEGG, 1998, 1999), nous complétons ici cette étude, par la description de trois espèces nouvelles se rapportant au genre Forcipomyia Meigen.

Forcipomyia pseudonigra n. sp. (fig. 1 à 13)

Mâle. - Holotype. Longueur totale approximative 2,2 $\mathrm{mm}$.

Tête. - Yeux nus, contigus sur toute la hauteur de leur bord interne. Antennes (fig. 4): scape ct pédicelle brun sombre; article III piriforme, beige clair uniforme; articles IV à XI, bien séparés, subégaux. progressivement rétrécis du premier jusqu'au dernier, beige clair avec une nette bande médiane plus claire, juste au-dessus de la ligne de soies formant le panache; article XII très long, à base renfléc, puis mince et cylindrique jusqu'à l'apex, beige clair, légèrement moucheté de brun sur toute sa longueur: article XIII cylindrique à base renflée, beige clair avec une bande claire juste au-dessus des soies basales disposées en verticille, moucheté de brun sur le reste du corps; article XIV subcylindrique, d'une coloration comparable à l'article précédent; article XV plus ou moins renflé, acuminé distalement et terminé par un mucron, beige clair uniforme, légèrement moucheté de brun. Article III avec des sensilla chaetica épars; articles IV-XII avec de longs sensilla chaetica disposés en verticille et formant le panache, l'article XII porte en outre des sensilla chaetica plus courts et vigoureux, disposés à intervalles réguliers, sur presque toute sa longueur; articles XIII-XIV avec respectivement 11 et 5 sensilla chaetica vigoureux implantés à la base et des sensilla trichodea et basiconica épars sur le reste du corps; article XV avec uniquement des sensilla trichodea et basiconica épars. Articles III-V avec 2 sensilla trichodea majeurs; articles IV-IX avec 1 sensillum trichodcum mineur et 1 à 2 sensilla basiconica. Mensurations: 79, 48, $46,44,44,44,46,46,48,213,128,77,99$. Longueur totale: $962 \mu \mathrm{m}$. Clypéus triangulaire à sommet postérieur tronqué, recouvert d'une fine pubescence et portant 8 soies fortes, disposées sur deux rangécs sur toute la hauteur de sa partie médiane. Pièces buccales: labre épipharynx et hypopharynx terminés en pointe finement pubescente; mandibules et maxilles inermes, à peine visibles; labium et labelles recouvrant presque entièrement les autres pièces. Palpe maxillaire (fig. 5) : article I trapu, à peine plus long que large, beige très clair, bruni sur son bord externe; article II subcylindrique, brun clair; article III long. fusiforme, brun clair, légèrement éclairci à son apex, présentant au tiers basal une petite fossette sensorielle circulaire, d'une profondeur modérée, ornée d'organes claviformes dépassant bien de l'ouverture: 

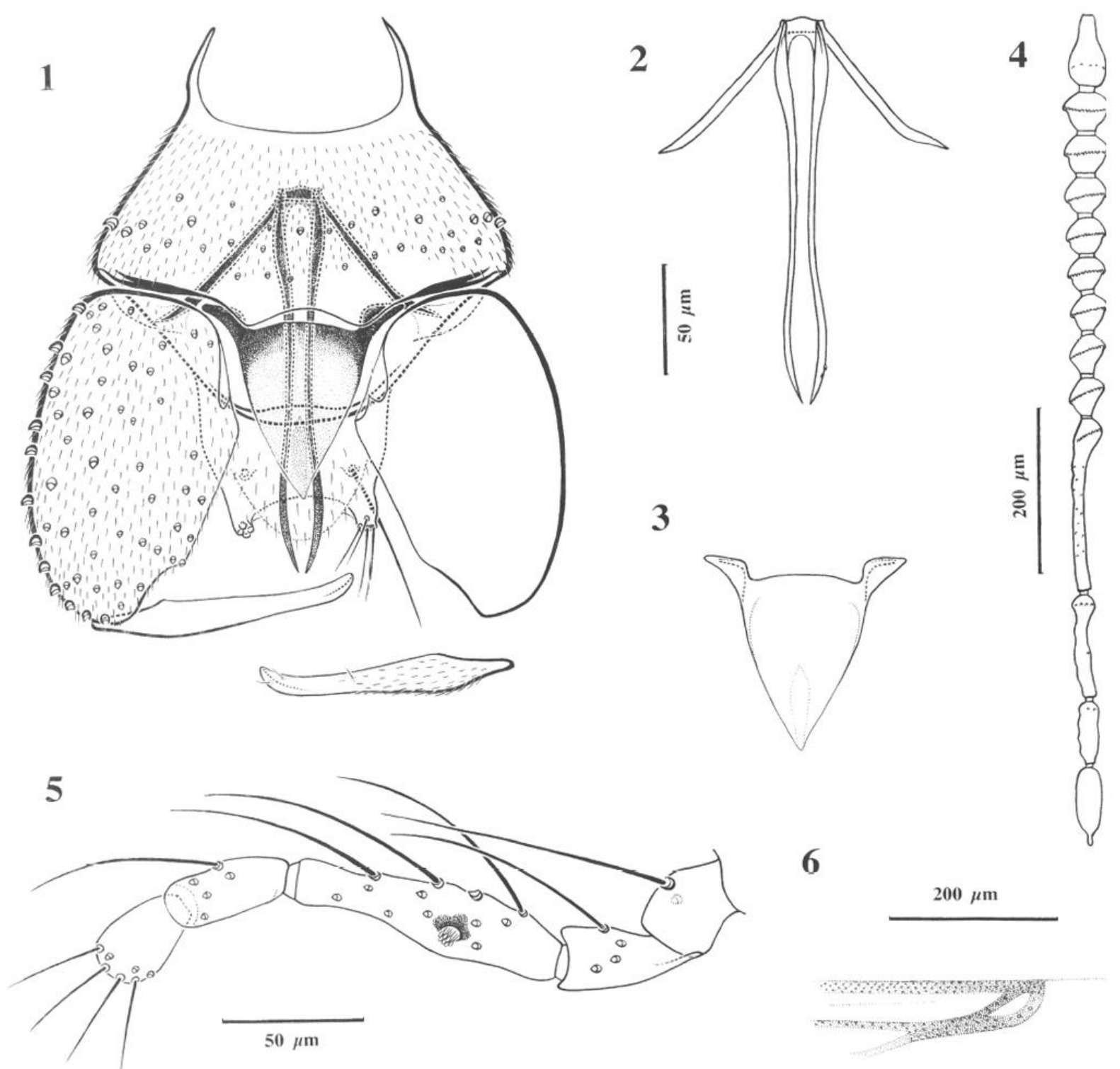

Fig. 1 à 6. - Forcipomyia pseudonigra n. sp.: $\delta$, holotype. - 1, Pince génitale en vue ventrale; -2 , Paramères el apodèmes dorsaux des coxites isolés; - 3, Ėdéage isolé; - 4, Antenne, forme générale; - 5, Palpe en vue ventrale: - 6. Détail des cellules radiales de l'aile.

article IV subcylindrique, brun clair, un peu éclairci à ses extrémités; article V élargi de la base à l'apex, un peu plus clair que l'article précédent. Mensurations : 35, 38, 103, 46, 40. Longueur totale : $262 \mu \mathrm{m}$.

Thorax. - Mésonotum, scutellum et métanotum uniformément brun sombre. Pilosité modérée, composée de soies faibles à fortes. Scutellum portant 15 soies fortes disposées en ligne transversale médiane et une quinzaine de soies de vigueur moyenne ou faible en avant et tout au long de cette ligne. Ailes claires, longues et étroites. Complexe radial légèrement bruni ; première cellule radiale courte et fermée, la seconde, de même longueur, bien ouverte et parfaitement arrondie sur son bord distal (fig. 6). Macrotriches abondants sur toute la surface alaire mais respectant cependant les cellules sous-costale et basale. Mensurations: I.: $1512 \mu \mathrm{m}, \mathrm{I}: 459 \mu \mathrm{m}$, costa: $594 \mu \mathrm{m}$, cubitale: $702 \mu \mathrm{m}$; le rapport alaire CR (longueur de la costa divisće par la longueur de l'aile), est de 0,39. Balanciers uniformément clairs. Pattes : fémurs, tibias et tarsomères des trois paires, cylindriques, d'un brun s'atténuant progressivement sur les tarsomères, arec un très net éclaircissement basal et distal sur les fémurs, les tibias et les tarsomères I-II, basal sur les tarsomères III-IV, donnant à l'ensemble des pattes un aspect plus ou moins annelé (fig. 12). Pilosité relativement dense, composée de soies courtes à longues dont certaines, vues à un fort grossissement. sont très finement barbelées. Les tarsomères I-IV des trois paires portent, en outre, des soies courtes et 


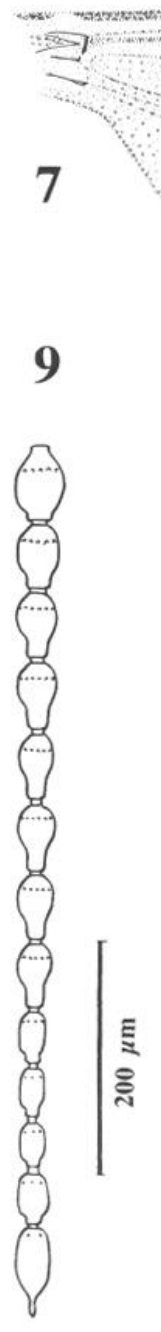

12
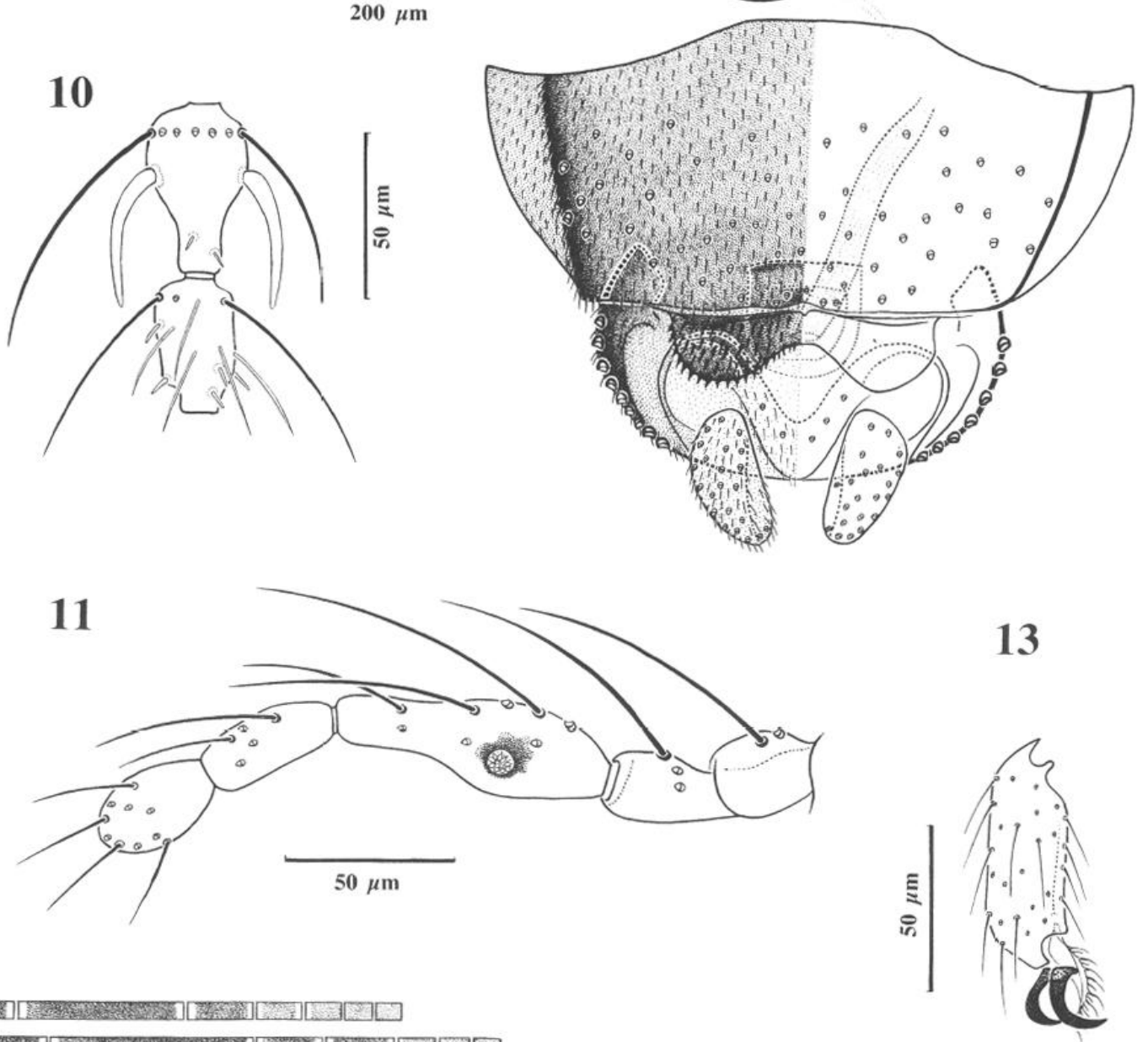

Fig. 7 à 13. - Forcipomyia pseudonigra n. sp.: $\&$, Allotype. - 7, Aile ; - 8, Partie postérieure de l'abdomen et spermathèques, en vue ventrale; - 9, Antenne, forme générale; - 10, Articles antennaires X-XI, vus à un plus fort grossissement; - 11, Palpe en vue ventrale; - 12, Représentation schématique de la coloration des trois paires de pattes ; - 13, tarsomère $\mathrm{V}$, griffes et empodium en vue latérale.

vigoureuses, clairsemées et décroissantes en nombre sur les tarsomères I-II, implantées à l'apex sur les tarsomères III-IV. Apex du tibia antérieur armé d'un éperon bien développé et d'un petit peigne. Apex du tibia médian sans éperon ni peigne, mais avec quclques soics particulièrement longues et vigoureuses. Apex du tibia postérieur avec un éperon bien développé et deux peignes, le plus grand formé de 6 dents de longueur sensiblement égale. Toutes les pattes se terminent de façon identique, par des griffes en crochet à extrémité parfois bifide, entre lesquelles on observe un empodium très développé.

$\begin{array}{lrrrrrrrr}\text { Mensurations }(\mu \mathrm{m}): & 511 & 521 & 202 & 157 & 120 & 97 & 75 & \text { TR }^{1}=1,28 \\ & 586 & 614 & 195 & 217 & 135 & 97 & 75 & \text { TR }=0,89 \\ & 632 & 688 & 202 & 255 & 157 & 105 & 82 & \text { TR }=0,79\end{array}$

${ }^{1}$ Le rapport tarsal TR est égal à la longueur du basitarse divisé par la longueur du second tarsomère. 
Abdomen. - Pince génitale (fig. 1) entièrement brunâtre. Sternite IX trapézoïdal; bord antérieur légèrement concave, présentant de chaque côté une longue pointe étroite, dirigée en avant et orientée à son extrémité vers l'axe du corps; bord postérieur concave dans son tiers médian; largement pubescent dans sa partie médiane et portant de nombreuses soies fortes, implantées en lignes transversales irrégulières. Tergite IX court, régulièrement arrondi et renforcé à son sommet; appendices apico-latéraux bien développés, munis de 4 soies chacun. Coxite long, gros et entièrement pubescent à l'exception du bord basal interne. Dististyle long, progressivement rétréci de la base à l'apex, à peine incurvé, et terminé par un petit bec arrondi; pubescent sur ses deux tiers basaux. Edéage (fig. 3) triangulaire, largement bruni à la base; bras basaux triangulaires, courts et forts, dirigés latéralement; la partie médiane du corps et la partie distale, en forme de pointe parfois tronquée, sont très claires et peu consistantes. Paramères (fig. 2) fortement brunis, unis à la basc par un petit pont transversal fort et net; les parties libres, en forme de longues tiges fines, atteignant presque l'extrémité des coxites, à peine grossies dans leur tiers basal et très légèrement dans leur tiers distal, étroitement séparées sur toute leur longueur, légèrement plus resserrés dans leur tiers médian, se terminent en pointes, qui selon l'angle d'observation peuvent être plus ou moins larges. Apodèmes dorsaux des coxites longs et étroits, rejoignant obliquement à l'apex la base des paramères.

Femelle. - Allotype. Longueur totale approximative $1,8 \mathrm{~mm}$.

Tête. - Yeux nus, contigus sur toute la hauteur de leur bord interne. Antennes (fig. 9): scape et pédicelle brun sombre, ornés chacun d'une quinzaine de sensilla chaetica; article III brun clair, à pédicule court très éclairci; articles IV-XIV d'un brun clair, légèrement assombri au-dessus du verticille, de forme dite en flacon (IV-X à col long, XI-XIV à col court); article XV long, atténué distalement et terminé par un mucron. Articles III-X avec un verticille basal formé d'une quinzaine de sensilla chaetica longs et vigoureux; articles XI-XV avec un verticille basal formé de 6 à 7 sensilla chaetica de longueur et vigueur moyennes; 2 sensilla trichodea majeurs, hypertrophiés sur III à X; 1 sensillum trichodeum mineur et 1 sensillum basiconicum sur IV à IX; 2 sensilla basiconica sur X; articles XI à XV avec des sensilla trichodea et basiconica épars. Mensurations : 64, 55, 55, 57, 57, 57, 55, 55, 44, 44, 42, 44, 75. Longueur totale : $704 \mu \mathrm{m}$. Rapport antennaire $(\mathrm{XI} / \mathrm{X})$ : 0,8. Clypéus portant 9 soies fortes, implantées sur toute la hauteur de sa partie médiane. Pièces buccales et palpe maxillaire (fig. 11) de forme et de coloration identiques à celles du mâle. L'article III du palpe, plus nettement renflé vers sa partie médiane présente une fossette sensorielle, légèrement plus profonde, remplie d'organes claviformes ne dépassant pas de l'ouverture. Mensurations : 29, 33, 86, 44, 37. Longueur totale : $229 \mu \mathrm{m}$.

Thorax. - Comparable dans l'ensemble à celui du mâle. Ailes (fig. 7) légèrement brunies sur toute la surface du limbe, plus fortement sur le complexe radial, sur la moitié antérieure de la cellule r 5 et sur toutes les nervures. Très faible éclaircissement à l'apex de la costa. Cellules radiales conformées comme celles du mâle. Macrotriches très abondants, longs et épais sur le complexe radial, plus courts et plus étroits sur le reste du limbe, absents des cellules sous costale et basale. Mensurations: L: 1260 $\mu \mathrm{m}, 1: 495 \mu \mathrm{m}$, costa : $465 \mu \mathrm{m}$, cubitale : $525 \mu \mathrm{m}$; rapport alaire CR: 0,36. Balanciers uniformément clairs. Pattes: coloration (fig. 12) identique à celle du mâle. Pilosité légèrement plus dense, avec quelques soies moyennes légèrement lancéolées, disposées d'une façon très irrégulière sur les trois paires. Le cinquième tarsomère (fig. 13) porte des griffes égales et un empodium très développé à toutes les pattes.

$\begin{array}{lrrrrrrrr}\text { Mensurations }(\mu \mathrm{m}): & 409 & 446 & 180 & 127 & 97 & 82 & 67 & \mathrm{TR}=1,41 \\ & 502 & 558 & 180 & 184 & 97 & 82 & 67 & \mathrm{TR}=0,97 \\ & 539 & 604 & 195 & 217 & 127 & 97 & 75 & \mathrm{TR}=0,89\end{array}$

Abdomen (fig. 8). - Brunâtre, nettement plus sombre sur les segments VIII-IX, très clair sur le segment $\mathrm{X}$. Pilosité modérée et moyennement longue sur tous les sternites, plus longue, plus vigoureuse et plus dense, d'avant en arrière, sur les pleures, particulièrement sur le segment IX. Sternite VIII deux fois aussi large que long, bord antérieur légèrement convexe dans son tiers médian, bord postérieur droit, entièrement couvert d'une fine pubescence, suivi de deux lobes bien individualisés (gonapophyses), couverts d'une pubescence vigoureuse. Sternite $\mathrm{X}$ en forme de $\mathrm{V}$, légèrement pubescent, portant latéralement 2 à 3 soies faibles. Sclérification génitale en forme de fenêtre, peu visible; portion distale de l'oviducte (vagin) large, longue et particulièrement très apparente. Deux spermathèques fonctionnelles, piriformes, subégales $(70 \times 55$ et $64 \times 46 \mu \mathrm{m})$ à petit col. 
Etymologie. - Le nom "pseudonigra" rappelle l'étroite ressemblance de cette espèce avec Forcipomyia nigra (Winnertz,1852), avec laquelle elle peut être facilement confondue.

Discussion. - Forcipomyia pseudonigra n. sp. est, par sa morphologie, très proche de F. nigra (Winnertz, 1852). Elle s'en distingue cependant aisément par les caractères propres suivants. Pour les deux sexes: par la coloration générale plus claire, par la coloration des pattes présentant, très nettement, des éclaircissements aux extrémités des fémurs, des tibias, des tarsomères I-III ainsi qu'à la base des tarsomères IV, et par la profondeur de la fossette sensorielle du palpe. Chez le mâle: par la forme des paramères dont les parties libres, en forme de longues tiges parallèles, sont très étroitement séparées, en particulier dans leur tiers médian. Chez la femelle: par la forme des articles antennaires et par le rapport antennaire $\mathrm{XI} / \mathrm{X}$, inférieur à 1 .

Holotype : $\delta$, Suisse, forêt du Sihlwald (hêtraie), altitude $560 \mathrm{~m}$, (TWT) du 24.V au 19.VI.1996; ALLOTYPE: + , idem, (TWT) du 25.IV au 23.V.1996, réc. K. Schiegg.

Autres spécimens: 19 , idem, (TWT) du 25.IV au 11.V.1996; 10, 2\%, idem, (TWT) du 13.VIII au

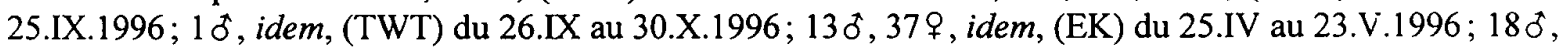
91 \&, idem, (EK) du 24.V au 19.VI.1996; $27 \delta^{\circ}, 59$, idem, (EK) du 20.VI au 18.VII.1996; $45 \delta^{\star}, 1999$, idem,

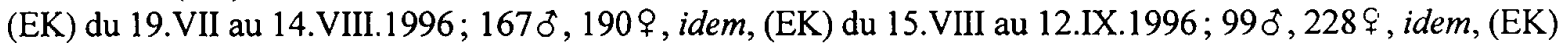
du 13.IX au 7.XI.1996; 50, 11 , idem, (EK) du 8.XI.1996 au 1.IV.1997; 9 , 19\%, idem, (EK) du 23.IV au 21.V.1997; $1 \delta^{\circ}, 16 \%$, idem, (EK) du 22.V au 20.VI.1997; 40, 7\%, idem, (EK) du 21.VI au 18.VII.1997; $70^{\star}$, 63 , idem, (EK) du 19.VII au 15.VIII.1997; 7 , 27\%, idem, (EK) du 16.VIII au 12.IX.1997; 60, 25\%, idem, (EK) du 13.IX au 6.XI.1997; 30 , 2 \%, idem, (TWT) du 19.VII au 31.VII.1997; réc. K. Schiegg.

Déposés au Musée Zoologique de l'Université Louis Pasteur et de la Ville de Strasbourg.

\section{Forcipomyia sihlwaldensis n. sp. (fig. 14 à 26)}

Mâle. - Holotype. Longueur totale approximative $2,3 \mathrm{~mm}$.

Tête. - Yeux nus, contigus sur toute la hauteur de leur bord interne. Antennes (fig. 14): scape et pédicelle brun sombre; article III piriforme, beige; articles IV-XI d'abord aussi longs que larges, puis progressivement plus étroits; articles XII-XIII renflés à la base, puis longuement cylindriques, le premier nettement plus long que le second; articles XIV-XV subcylindriques, le dernier acuminé distalement et terminé par un mucron; articles IV-VI bien séparés, beige clair avec une bande plus claire juste au-dessus de la ligne de soies formant le panache; articles VII-X étroitement soudés, beige clair progressivement uniforme; article XI bien individualisé, beige clair; articles XII-XV beige clair, mouchetés de brun. Article III avec une quinzaine de sensilla chaetica d'une longueur moyenne; articles IV-XII avec de longs sensilla chaetica disposés en verticille formant le panache; l'article XII porte en outre de nombreux sensilla chaetica courts, disposés irrégulièrement sur presque toute sa longueur; article XIII avec une dizaine de sensilla chaetica disposés en verticille basal et 4 à 5 plus faibles sur la moitié distale entremêlés à quelques sensilla trichodea et basiconica épars; article XIV avec 7 à 8 sensilla chaetica implantés à la base et des sensilla trichodea et basiconica épars sur le reste du corps; article XV avec de nombreux sensilla trichodea et basiconica épars. Articles III-V avec 2 sensilla trichodea majeurs; article VI avec 1 sensillum trichodeum majeur; articles IV-IX avec 1 sensillum trichodeum mineur; articles IV-X avec 1 sensillum basiconicum. Mensurations: 88, 48, 46, 46, 44, 44, 42, 48, 51, 222, 130, 84, 117. Longueur totale: $1010 \mu \mathrm{m}$. Clypéus en forme d'écusson, entièrement pubescent, portant 6 soies fortes alignées par paires dans sa partie médiane. Pièces buccales et palpes maxillaires (fig. 15), comparables à ceux du mâle de F. pseudonigra. Mensurations du palpe : 27, 37, 108, 48, 31. Longueur totale : $251 \mu \mathrm{m}$.

Thorax. - Mésonotum, scutellum et métanotum uniformément bruns. Pilosité modérée, composée de soies d'une longueur moyenne. Scutellum avec 12 soies fortes disposées en ligne transversale et 6 soies plus faibles en avant, réparties dans la partie médiane. Ailes très claires; cellules radiales et partie distale de la nervure R4 fortement brunies. Première cellule radiale courte et fermée, la seconde bien ouverte, plus ou moins en forme de losange; $\mathrm{R} 2+3$ légèrement coudée avant sa fusion avec la costa, cette dernière se prolonge très faiblement au-delà de la fusion (fig. 19). Macrotriches abondants sur tout le limbe, y compris 

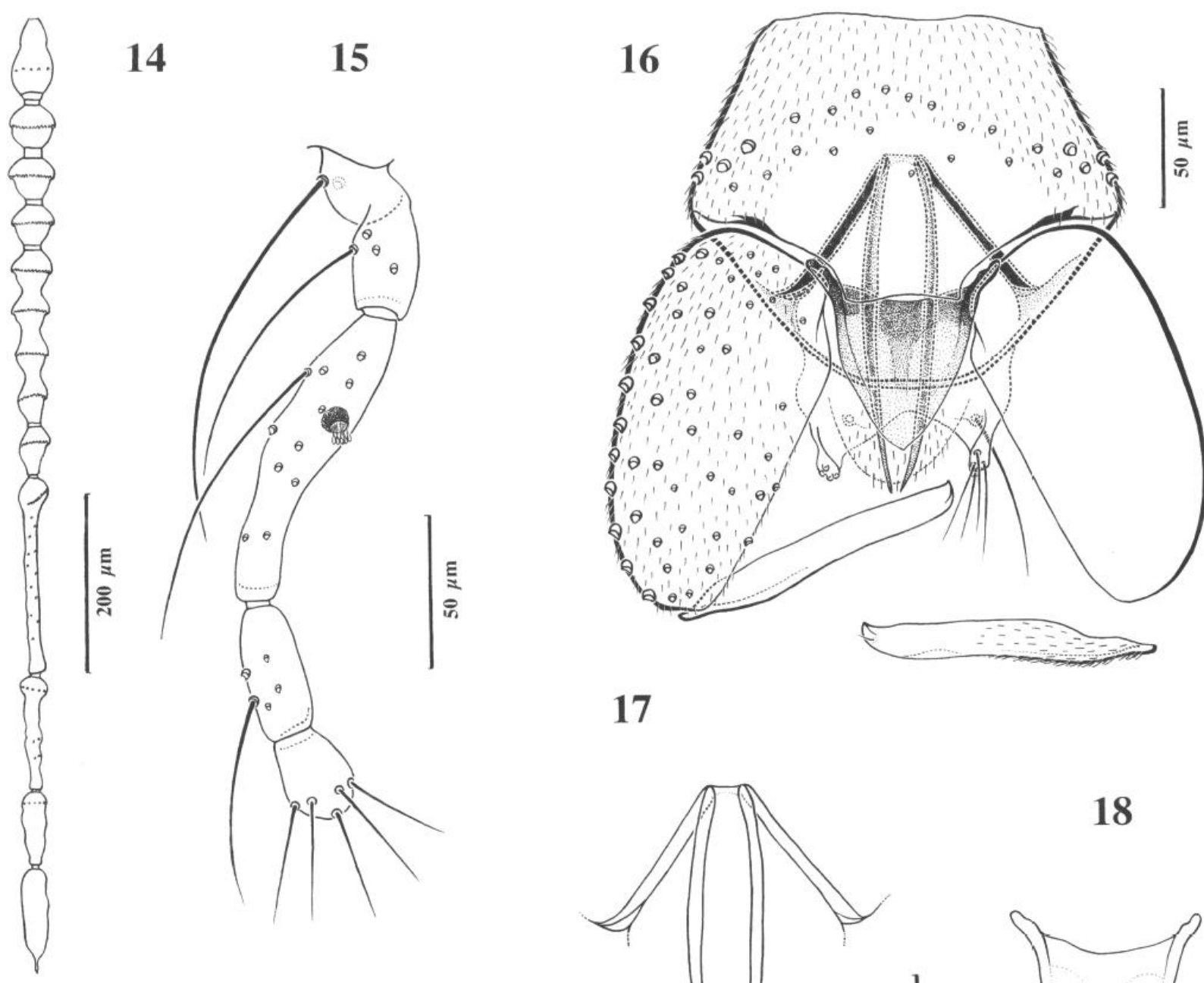

17

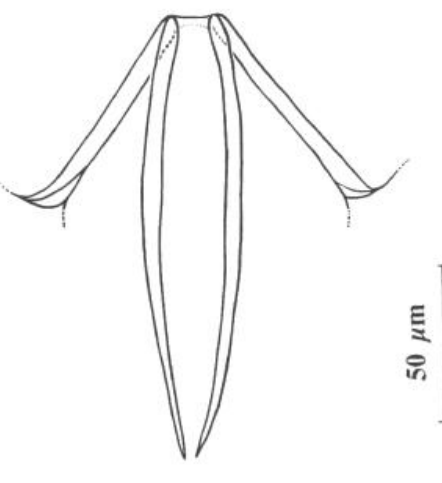

18

19

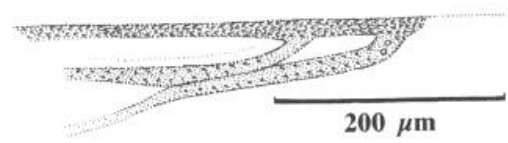

Fig. 14 à 19. - Forcipomyia sihlwaldensis n. sp. : $\delta$, holotype. - 14, Antenne, forme générale; - 15, Palpe en vue ventrale; - 16, Pince génitale en vue ventrale; - 17, Paramères et apodèmes dorsaux des coxites isolés; - 18, Edéage isolé ; - 19, Détail des cellules radiales de l'aile.

dans la cellule basale, plus rares dans la cellule sous-costale. Mensurations: $\mathrm{L}: 1539 \mu \mathrm{m}, 1: 459 \mu \mathrm{m}$, costa: $675 \mu \mathrm{m}$, cubitale : $729 \mu \mathrm{m}$; rapport alaire CR: 0,43. Balanciers uniformément clairs. Pattes : pour les trois paires, fémurs, tibias et basitarses d'un brun clair avec un net éclaircissement à leurs extrémités; tarsomères II-V plus ou moins éclaircis à leur base (fig. 25). Nombreuses soies moyennes à longues, très finement barbelées distalement, sur i'ensemble des segments; les tarsomères I-IV portent en outre des soies courtes et fortes, éparses et décroissantes en nombre sur I-II, disposées à l'apex sur III-IV, ces dernières étant plus vigoureuses sur les tarsomères médians. Apex des tibias conformes, dans l'ensemble, à ceux de $F$. pseudonigra. Grand peigne tibial postérieur à 8 dents. Griffes simples en forme de crochet. Empodia très développés.

$\begin{array}{lllllllll}\text { Mensurations }(\mu \mathrm{m}): & 547 & 585 & 240 & 187 & 135 & 105 & 75 & \mathrm{TR}=1,28 \\ & 645 & 705 & 210 & 240 & 142 & 97 & 75 & \text { TR }=0,87 \\ & 697 & 705 & 225 & 285 & 172 & 112 & 75 & \text { TR }=0,78\end{array}$

Abdomen. - Pince génitale (fig. 16) brunâtre; dististyles clairs, légèrement brunis à l'apex. Sternite IX trapézoïdal; bord antérieur droit; bord postérieur surélevé dans son tiers médian; pubescent sur sa moitié antérieure et sur ses bords latéraux, portant de nombreuses soies fortes disposées sur deux rangées irrégulières et transversales, largement dénudé dans sa partie médiane postérieure. Tergite IX court, régu- 

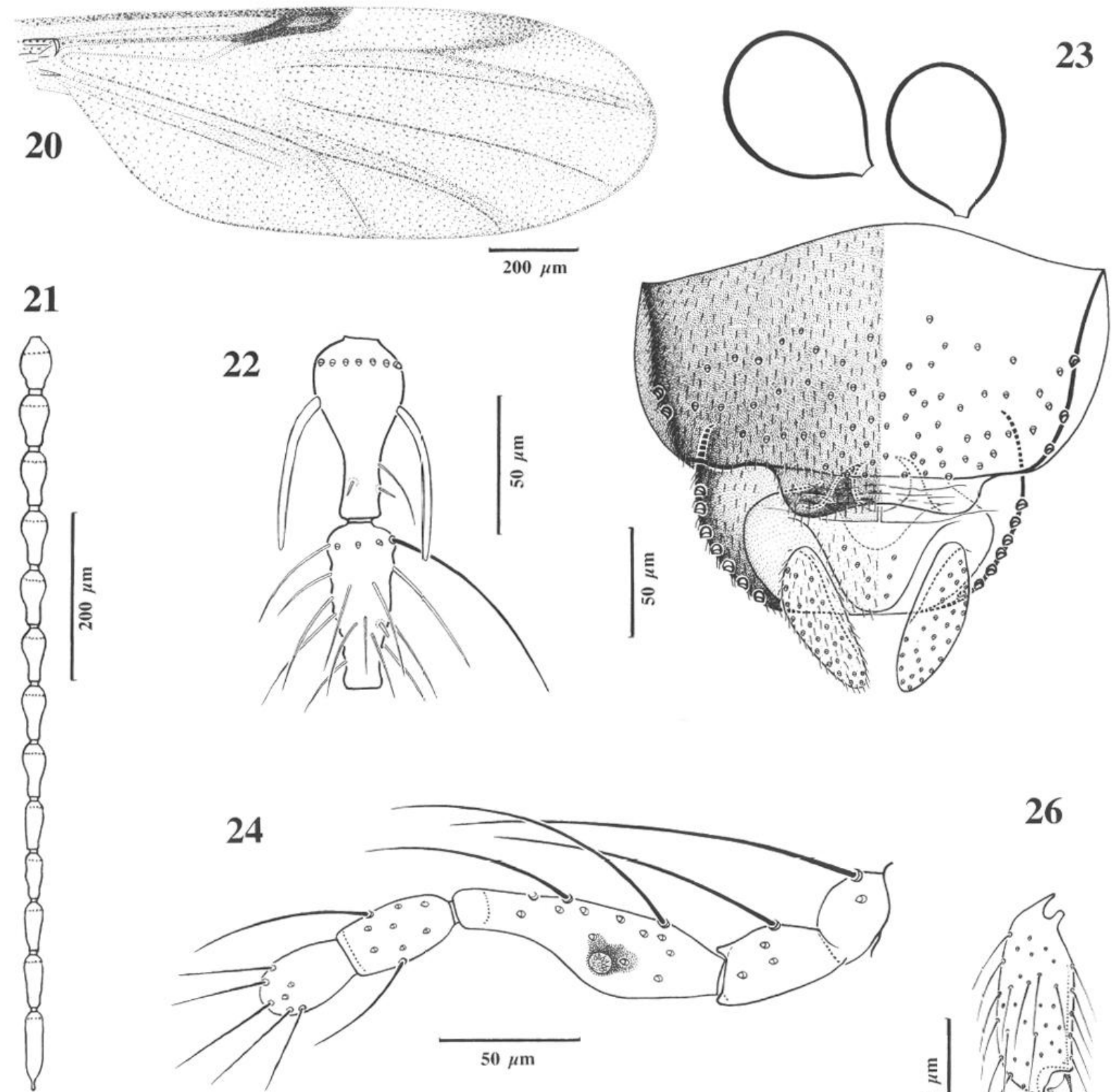

22
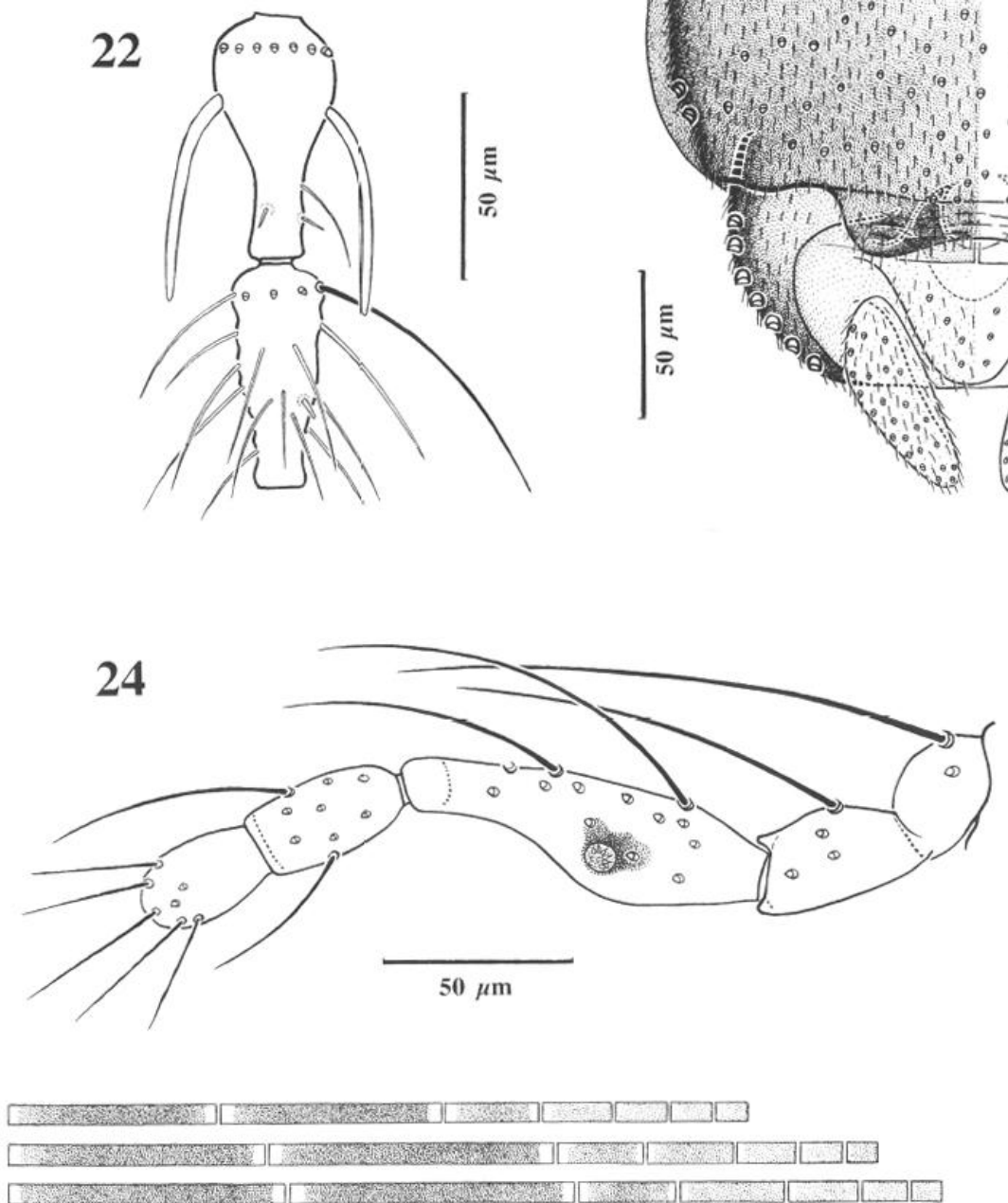

Fig. 20 à 26. - Forcipomyia sihlwaldensis n. sp.: $\$$, allotype. - 20, Aile; - 21, Antenne, forme générale; - 22, Articles antennaires X-XI, vus à un plus fort grossissement; - 23, Partie postérieure de l'abdomen et spermathèques, en vue ventrale; -24 , Palpe en vue ventrale; -25 , Représentation schématique de la coloration des trois paires de pattes; -26 , Tarsomère $V$, griffes et empodium en vue latérale.

lièrement arrondi et renforcé à son sommet; appendices apico-latéraux portant 4 soies chacun. Coxite long, entièrement pubescent à l'exception d'une large plage basale interne. Dististyle long, conservant à peu près le même diamètre sur toute sa longueur, se terminant en forme de bec à son extrémité; pubescent sur toute sa moitié basale. Edéage (fig. 18) triangulaire; largement bruni à la base, s'estompant progressivement dans l'axe du corps et sur les côtés latéraux, clair sur le reste; sommet postérieur plus ou moins arrondi et peu consistant ; bras basaux courts et cylindriques, à peine courbés et dirigés dorsalement. Paramères (fig. 17) brun clair, unis entre eux à la base par une suture transversale étroite, parfois peu apparente, les parties libres relativement longues, atteignant les deux tiers du coxite, régulièrement rétrécies de la base 
à l'apex, légèrement arquées vers l'intérieur, se terminent en pointes fines. Apodèmes dorsaux des coxites noirâtres, longs et cylindriques, dirigés obliquement pour rejoindre à l'apex, la base des paramères.

Femelle. - Allotype. Longueur totale approximative $2 \mathrm{~mm}$.

Tête. - Yeux nus, contigus sur toute la hauteur de leur bord interne. Antennes (fig. 21): scape et pédicelle brun, portant chacun une douzaine de sensilla chaetica; article III brun clair, à pédicule court et clair; articles IV-X uniformément brun clair, nettement plus longs que larges, de forme dite en flacon évasé; articles XI-XV subcylindriques, brun clair, mouchetés de brun, le dernier acuminé distalement et terminé par un mucron. Verticille basal formé d'une quinzaine de sensilla chaetica plus ou moins vigoureux sur les articles III-X, 6 à 8 plus faibles sur les articles XI-XV; 2 sensilla trichodea majeurs, bien développés, sur III-X; 1 sensillum trichodeum mineur sur IV-IX; 1 sensillum basiconicum sur IV-IX; 2 à 3 sur III et X; des sensilla trichodea et basiconica épars sur XI-XV. Mensurations: 66,68 , $68,70,68,68,68,68,62,62,59,64,101$. Longueur totale : $892 \mu \mathrm{m}$. Rapport antennaire $(\mathrm{XI} / \mathrm{X}): 0,91$. Clypéus et pièces buccales identiques à ceux du mâle. Palpe maxillaire (fig. 24) d'une coloration comparable à celle du mâle. L'article III, plus fortement renflé vers sa partie médiane, présente une fossette sensorielle d'un diamètre et d'une profondeur plus amples, munie d'organes claviformes ne dépassant pas de l'ouverture. Mensurations: 29, 46,101, 44, 31. Longueur totale : $251 \mu \mathrm{m}$.

Thorax. - Identique à celui du mâle. Ailes (fig. 20) légèrement teintées de brun sur toute la surface du limbe; fortement brunie sur les cellules radiales et dans la partie médiane de la cellule $\mathrm{r} 5$, dans l'angle formé par la nervure R4 et le bord antérieur de l'aile, plus légèrement sur toutes les nervures; large éclaircissement à l'apex de la costa et dans la partie distale de la cellule $r 5$. Cellules radiales comparables à celles de l'aile du mâle (fig. 19). Macrotriches très abondants dans toutes les cellules. Mensurations: $\mathrm{L}: 1350 \mu \mathrm{m}, \mathrm{l}: 540 \mu \mathrm{m}$, costa : $660 \mu \mathrm{m}$, cubitale : $615 \mu \mathrm{m}$; rapport alaire CR : 0,48. Balanciers uniformément clairs. Pattes (fig. 25) coloration et pilosité comparables à celles du mâle. Grand peigne tibial postérieur à 7 dents. Griffes (fig. 26) simples, égales, en forme de crochet. Empodia bien développé.

$\begin{array}{lllllllll}\text { Mensurations }(\mu \mathrm{m}): & 510 & 562 & 232 & 172 & 127 & 105 & 75 & \mathrm{TR}=1,34 \\ & 637 & 712 & 217 & 217 & 142 & 105 & 75 & \mathrm{TR}=1,00 \\ & 682 & 712 & 240 & 262 & 172 & 112 & 75 & \mathrm{TR}=0,91\end{array}$

Abdomen (fig. 23). - Brunâtre, plus sombre sur les segments VIII-IX. Pilosité relativement dense, en particulier sur les pleures. Sternite VIII rectangulaire, bord antérieur progressivement lobé en son milieu, bord postérieur droit, suivi de deux lobes (gonapophyses) peu développés, déprimés, marqués de rides transversales et couverts d'une pubescence peu vigoureuse. SterniteX portant de chaque côté un alignement de 3 à 4 soies d'une longueur moyenne. Sclérification génitale peu apparente, droite et étroitement fendue au milieu dans sa partie postérieure, prolongée en pointes courbes et convergentes en profondeur dans sa partie antérieure; oviducte non apparent. Deux spermathèques fonctionnelles, piriformes, subégales $(81 \times 62$ et $73 \times 55)$ à petit col.

Etymologie. - "sihlwaldensis" se réfère au nom de la forêt dans laquelle l'espèce a été capturée.

Discussion. - Par certains de ses caractères, Forcipomyia sihlwaldensis n. sp. se rapproche de $F$. brevipedicillata (Kieffer, 1901) et de $F$. brevipennis (Macquart, 1826). Elle s'en différencie cependant aisément par les caractères propres suivants : pour les deux sexes, par la coloration des ailes et des pattes; chez le mâle, par le rapport des longueurs des articles XII/XIII des antennes, par la forme de l'édéage et des paramères; chez la femelle, par la forme des articles proximaux des antennes.

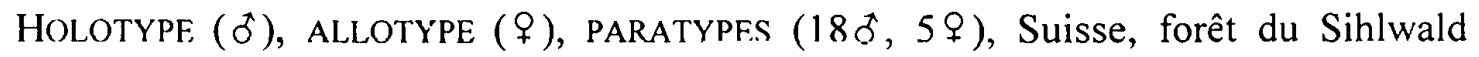
(hêtraie), altitude $560 \mathrm{~m}$, (EK) du 24.V au 19.VI.1996, réc. K. Schiegg.

Autres spécimens: $2 \delta, 9 q$, idem, (EK) du 20.VI au 18.VII.1996; 7q, idem, (EK) du 19.VII au 14.VIII.1996; $1 \delta^{\delta}$, idem, (EK) du 23.IV au 21.V.1997; 12 $\delta^{\circ}, 10 \%$, idem, (EK) du 22.V au 20.VI.1997; $3 \delta$. 19 9 , idem, (EK) du 21.VI au 18.VII.1997; 7q, idem, (EK) du 19.VII au 15.VIII.1997; 3ठ, idem, (EK) du 16.VIII au 12.IX.1997; réc. K. Schiegg.

Déposés au Musée Zoologique de l'Université Louis Pasteur et de la Ville de Strasbourg. 
Forcipomyia helvetica n. sp. (fig. 27 à 40)

Mâle. - Holotype. Longueur totale approximative $2,8 \mathrm{~mm}$.

Tête. - Yeux soudés, non pubescents. Antennes (fig. 27): scape et pédicelle brun; article III piriforme, brun clair; articles IV à XI, bien séparés, d'abord aussi longs que larges puis progressivement p'us longs que larges, passant d'un brun clair avec une bande plus claire au-dessus du verticille à un beige clair plus ou moins uniforme; article XII semblable aux précédents à la base, puis longuement cylindrique jusqu'à l'apex, d'un beige clair très légèrement moucheté de brun; articles XIII-XV subcylindriques, nettement mouchetés de brun sur toute la surface de leur corps, le dernier acuminé distalement et terminé par un mucron. Article III avec une quinzaine de sensilla chaetica d'une longueur moyenne; articles IV-XII avec de longs sensilla disposés en verticille et formant le panache, l'article XII porte en outre, à l'exclusion de tout autres sensilles, des sensilla chaetica courts disposés régulièrement sur presque toute sa longueur; article XIII avec un verticille basal formé d'une douzaine de sensilla chaetica et sur le reste du corps des sensilla chaetica, trichodea et basiconica épars; article XIV avec une dizaine de sensilla chaetica disposés en verticille à la base et des sensilla trichodea et basiconica épars sur le reste du corps; article XV avec uniquement des sensilla trichodea et basiconica épars. Articles III-VI avec 2 sensilla trichodea majeurs; articles IV-IX avec 1 sensillum trichodeum mineur; articles IV-X avec 1 à 3 sensilla basiconica. Mensurations: 108, 64, 62, 62, $57,57,57,62,62,279,143,99,150$. Longueur totale: $1262 \mu \mathrm{m}$. Clypéus en forme d'écusson, entièrement pubescent, portant 9 soies fortes médianes. Pièces buccales: labre épipharynx terminé en pointe finement pubescente; hypopharynx terminé en pointe lisse; mandibules inermes; maxilles terminées en pointe, portant sur la partie distale de leur bord externe 7 à 8 éléments d'une pubescence, relativement longue et fine; labium et labelles recouvrant entièrement les autres pièces. Palpe maxillaire (fig. 28), brun clair avec un léger éclaircissement aux articulations; articles I-II subcylindriques; article III cylindrique, légèrement grossi dans sa moitié basale, présentant, juste au-dessous du milieu du bord interne, une petite fossette sensorielle peu profonde, ornée d'organes en forme de baguettes de tambour; articles IV-V cylindriques. Mensurations : $35,49,136,64,48$. Longueur totale : $332 \mu \mathrm{m}$.

Thorax. - Mésonotum brun, légèrement éclairci sur les épaules; scutellum brun, légèrement jaunâtre sur son bord antérieur; métanotum uniformément brun sombre; pleures bruns, à l'exception d'une large bande dorsale jaunâtre, longeant le mésonotum sur toute sa longueur. Pilosité modérée. Scutellum portant une quarantaine de soies faibles à fortes, disposées d'avant en arrière, sur plusieurs lignes transversales irrégulières. Ailes longues et étroites, limbe d'un teint jaunâtre, légèrement bruni sur les cellules radiales, dans la cellule r5 sur la nervure R4, et plus discrètement sur les autres nervures. Première cellule radiale fermée, la seconde bien ouverte, de forme plus ou moins quadrangulaire (fig. 29). Macrotriches abondants sur toute la surface du limbe à l'exception de la cellule sous-costale où ils sont absents. Mensurations : L: $1917 \mu \mathrm{m}, 1: 567 \mu \mathrm{m}$, costa : $918 \mu \mathrm{m}$, cubitale : $864 \mu \mathrm{m}$; rapport alaire CR: 0,47. Balanciers uniformément clairs. Pattes, pour les trois paires : fémurs, tibias et tarsomères cylindriques, d'un jaune pâle uniforme. Pilosité dense, relativement longue et concolore. Les tarsomères I-IV des trois paires portent, en plus, des soies courtes, vigoureuses et brunes, clairsemées et décroissantes en nombre sur les tarsomères I-II, disposées à l'apex sur les tarsomères III-IV. Apex du tibia antérieur avec un éperon et un petit peigne à peine visible. Apex du tibia médian sans éperon ni peigne. Apex du tibia postérieur avec un éperon et deux peignes, le plus grand formé de 9 dents dont les longueurs sont: $48,46,44,46,48,53,57,68,68$.Toutes les pattes se terminent par des griffes en crochet à extrémité parfois bifide, entre lesquelles on observe un empodium très développé.

$\begin{array}{lllllllll}\text { Mensurations }(\mu \mathrm{m}): & 651 & 642 & 225 & 292 & 195 & 142 & 97 & \text { TR }=0,77 \\ & 753 & 753 & 187 & 352 & 195 & 150 & 105 & \text { TR }=0,53 \\ & 828 & 828 & 210 & 367 & 232 & 165 & 105 & \text { TR }=0,57\end{array}$

Abdomen. - Pince génitale (fig. 30) brunâtre, plus ou moins éclaircie à l'avant du sternite IX et sur les dististyles. Sternite IX rectangulaire; bord antérieur légèrement concave, présentant de chaque côté une petite pointe triangulaire; bord postérieur nettement lobé dans son tiers médian; largement couvert d'une fine pubescence dans sa partie antérieure et portant de nombreuses soies fortes disposées en lignes transversales irrégulières médianes. Tergite IX court, régulièrement arrondi et renforcé à son sommet; appendices apico-latéraux portant 4 soies chacun. Coxite long, entièrement pubescent à l'exception d'une large plage basale interne. Dististyle droit, pubescent sur la moitié basale, progressivement rétréci de la base à l'extrémité, terminé par un petit bec arrondi. Edéage (fig. 32) en forme d'Y, entièrement et fortement bruni; bras basaux légèrement courbés et dirigés latéralement; bords latéraux en forme d'ailerons étroits, légèrement repliés dorsalement; partie distale en forme de forte pointe arrondie. Paramères (fig. 31) 

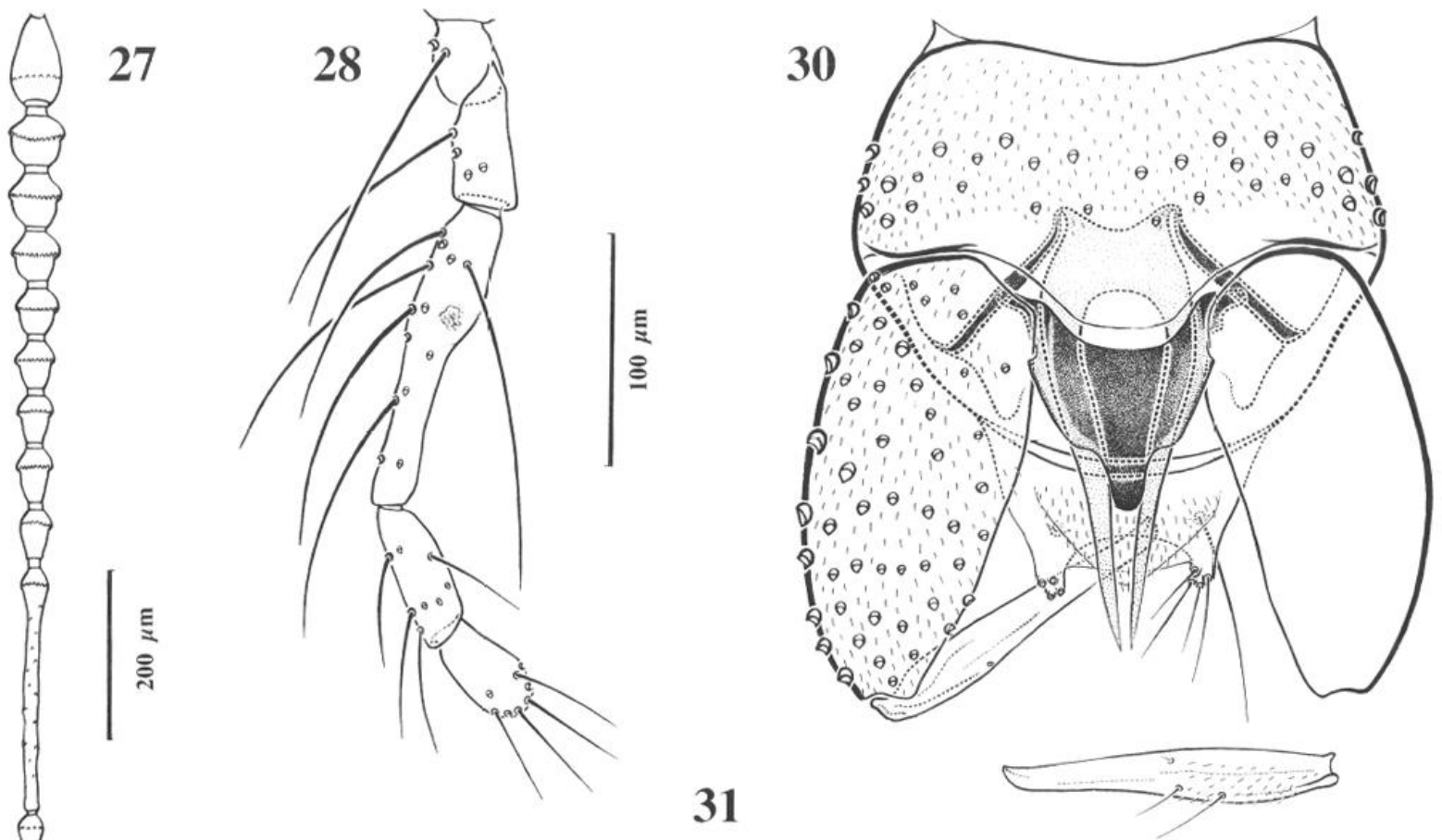

31
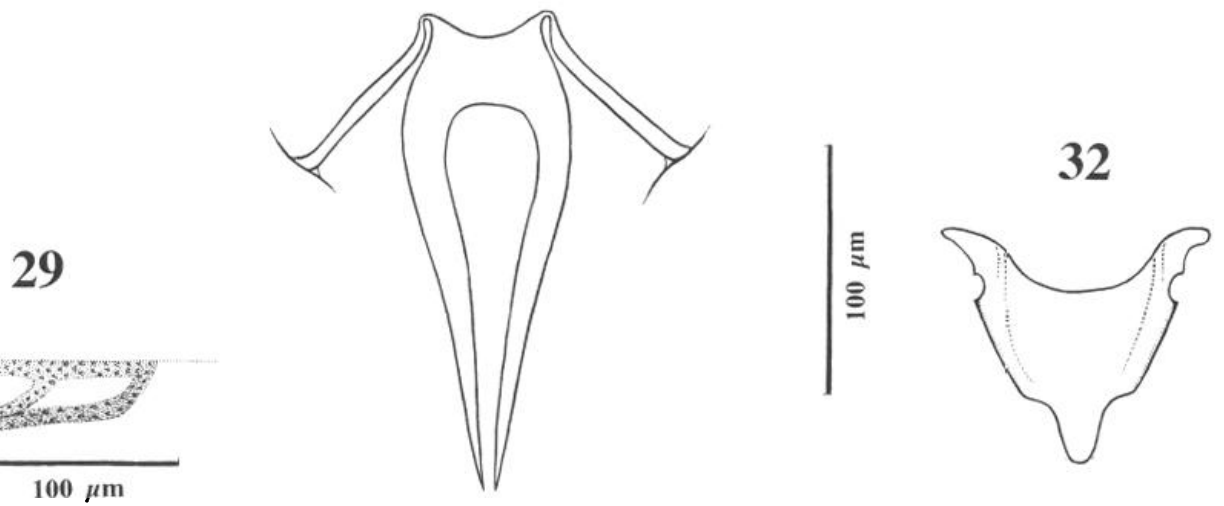

Fig. 27 à 32. - Forcipomyia helvetica $n$. sp : $\delta$, holotype. - 27, Antenne, forme générale ; - 28, Palpe en vue ventrale; -29 , Détail des cellules radiales de l'aile; -30 , Pince génitale en vue ventrale; - 31, Paramères et apodèmes dorsaux des coxites isolés; - 32, Edéage isolé.

jaunâtres, largement soudés à la base, les branches libres, courbes et larges à la base, progressivement et régulièrement rétrécies, convergentes à leur extrémité qui est acérée. Apodèmes dorsaux des coxites brunâtres, longs, amincis et recourbés à leur extrémité qui est très étroitement reliée à la base des paramères.

Femelle. - Allotype. Longueur totale approximative 2,8 mm.

Tête. - Yeux nus, contigus sur toute la hauteur de leur bord interne. Antennes (fig. 34): scape et pédicelle brun jaunâtre, portant respectivement une vingtaine et une dizaine de sensilla chaetica; articles III-X brun pâle, légèrement jaunâtres; articles XI-XV de la même teinte que les précédents mais nettement mouchetés de brun sur presque toute la surface du corps. Article III à pédicule court; articlcs IV-X subcylindriques, progressivement allongés et prenant légèrement la forme dite en flacons évasés; articles XI$\mathrm{XV}$ longs, cylindriques, le dernier acuminé distalement et terminé par un mucron. Tous les articles portent un verticille basal formé de sensilla chaetica vigoureux et bruns: 12 à 15 sur les articles III-X, 4 à 5 sur les articles XI-XIV et 2 sur XV. Articles III-X avec 2 sensilla trichodea majeurs, 1 à 2 mineurs et 2 à 4 sensilla basiconica; articles XI-XV avec de nombreux sensilla trichodea et basiconica épars. Mensurations: $68,57,59,59,66,68,68,68,92,90,92,97,134$. Longueur totale: $1018 \mu \mathrm{m}$. Rapport antennaire $(\mathrm{XI} / \mathrm{X}): 1,35$. Clypéus (fig. 33) entièrement pubescent, avec 12 soies fortes. Pièces buccales: labre épipharynx et hypopharynx terminés en pointe large, arrondie et inerme; mandibules armées sur la partie 


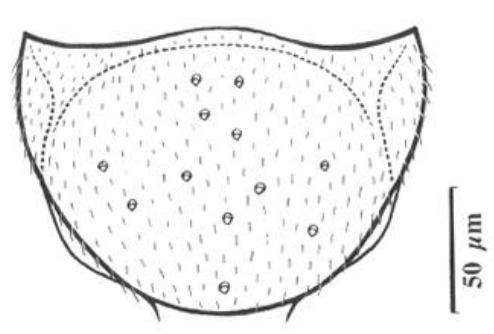

34

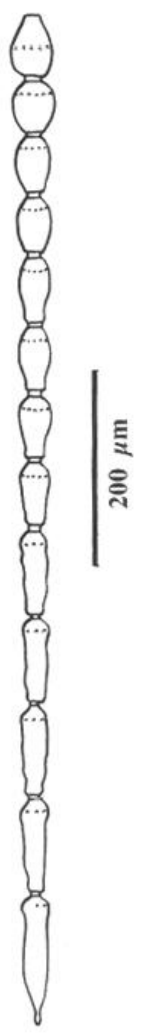

35

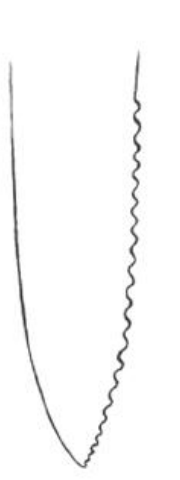

36

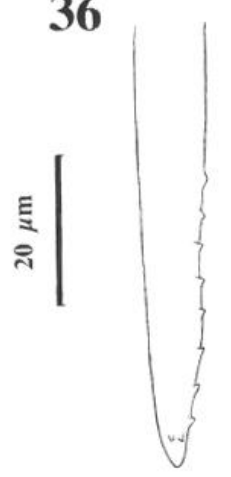

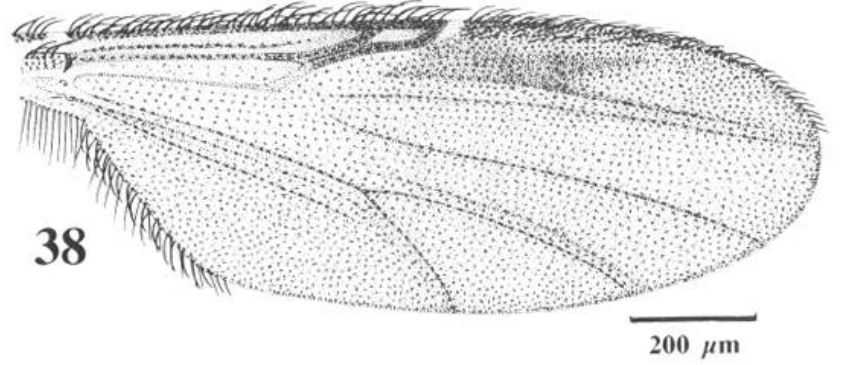

39

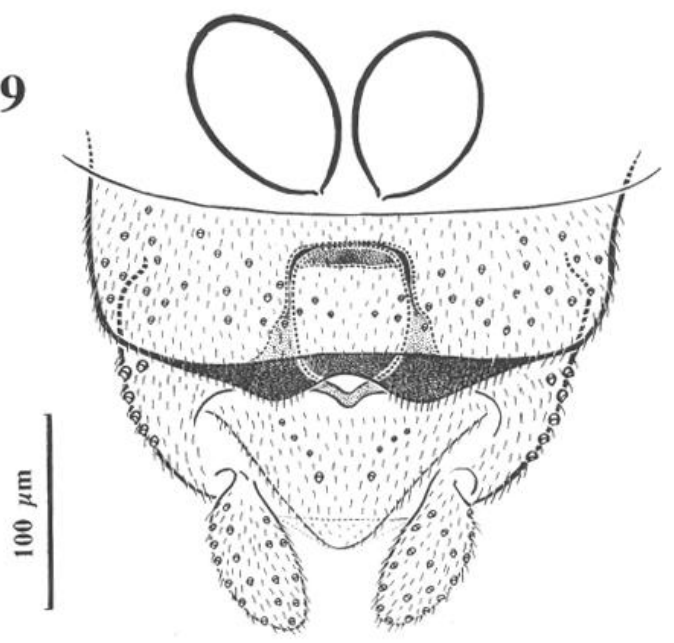

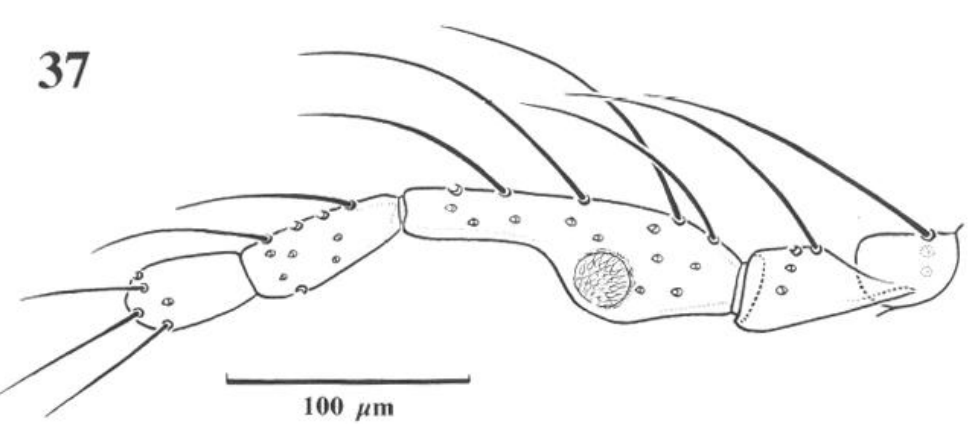

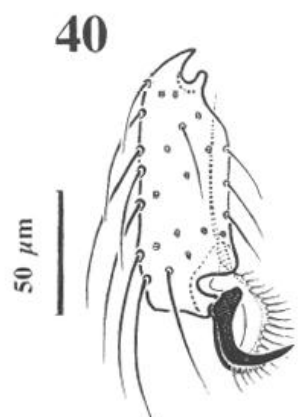

Fig. 33 à 40. - Forcipomyia helvetica n. sp: 9 , allotype. - 33, Clypéus; - 34, Antenne, forme générale ; - 35, 36, Extrémité distale de la mandibule et distale de la maxille; -37 , Palpe, vue ventrale; -38 , Aile; - 39, Partie postérieure de l'abdomen et spermathèques, en vue ventrale ; -40 , Tarsomère $\mathrm{V}$, griffes et empodium en vue latérale.

distale de leur bord interne d'une vingtaine de denticules de taille décroissante de la base à l'apex (fig. $35)$; maxilles armées sur la partie distale de leur bord externe d'une dizaine de très petites dents bien espacées (fig. 36); labium et labelles recouvrant entièrement l'ensemble des pièces buccales. Palpe maxillaire (fig. 37) d'une coloration identique à celui du mâle; articles I-II subcylindriques; article III renflé dans sa moitié basale, plus fortement vers la partie médiane de son bord interne où on observe une large fossette sensorielle, circulaire, de très faible profondeur, ornée d'organes claviformes dépassant à peine de l'ouverture; moitié distale étroite et cylindrique; articles IV-V subcylindriques, le dernier arrondi à l'apex. Mensurations : 42, 48, 141,68, 51. Longueur totale : $350 \mu \mathrm{m}$.

Thorax. - Coloration identique dans l'ensemble à celui du mâle; pilosité légèrement plus dense. Ailes (fig. 38) larges, relativement sombres sur toute la surface alaire, plus fortement brunies sur les cellules radiales ainsi que dans la cellule $r 5$, au-dessus de la nervure $\mathrm{R} 4$. Macrotriches très abondants, légèrement épaissis et fortement pigmentés sur le complexe radial, moins larges et plus clairs sur le reste du limbe. Mensurations : $\mathrm{L}: 1890 \mu \mathrm{m}, 1: 756 \mu \mathrm{m}$, costa: $945 \mu \mathrm{m}$, cubitale : $810 \mu \mathrm{m}$; rapport alaire CR: 0,50 . Balanciers uniformément clairs. Pattes: coloration et pilosité identiques à celles du mâle. Grand 
peigne tibial postérieur à 9 dents dont les longueurs sont: 44, 46, 44, 44, 44, 44, 51, 66, 73. Griffes (fig. 40) égales, courbes, en forme de crochet à toutes les pattes. Empodia très dévcloppés.

$\begin{array}{llllllllll}\text { Mensurations: } & 632 & 660 & 240 & 270 & 172 & 135 & 105 & \text { TR }=0,88 \\ & 772 & 865 & 217 & 345 & 180 & 142 & 105 & \text { TR }=0,62 \\ & 856 & 902 & 255 & 375 & 217 & 165 & 105 & \text { TR }=0,68\end{array}$

Abdomen (fig. 39). - Brun jaunâtre, légèrement plus clair sur les derniers segments : cerques jaunâtres. Pilosité dense, brune sur les sternites, alternativement brune et jaune sur les pleures des segments II à V, brune sur les pleures des segments suivants et jaune sur les cerques. Sternite VIII plus large que long, entièrement pubescent et portant de chaque côté une vingtaine de soies fortes, assez. largement séparées de leurs homologues dans l'axe du corps; bord antérieur plus ou moins droit ; bord postérieur à légère concavité médiane, suivi par deux lobes médiocres (gonapophyses). bien pigmentés et couverts d'une fine pubescence. Sternite $\mathrm{X}$ avec 2 soies postérieures fortes, bien séparées l'une de l'autre, et 3 à 4 plus faibles de chaque côté en avant des précédentes. Sclérification génitale en forme d'arche un peu plus haute que large; bord antérieur fort et transversal; bord postérieur plus étroit, légèrement en pointe; bords latéro-distaux élargis et prolongés sur les côtés. Deux spermathèques fonctionnelles de grande taille, subégales $(114 \times 66$ et $97 \times 57)$ à col très court.

Etymologie. $-F$. helvetica a été trouvé en Suisse $=$ Helvetia, d'où le nom de la nouvelle espèce.

Discussion. - Forcipomyia helvetica n. sp. se rapproche par ses caractères de $F$. suberis Clastrier, 1956 et de $F$. cirrhosa Clastrier, Rioux \& Descous, 1961. Elle s'en sépare cependant sans difficulté par l'association des caractères suivants. Chez les deux sexes : par la coloration des pattes et par la faible profondeur de la fossette sensorielle du palpe. Chez le mâle seulement: par la forme et la coloration de l'édéage.

Holotype ( $\delta$ ), allotype ( $q$ ), paratypes $(3 \delta, 5 q)$, Suisse, forêt du Sihlwald (hêtraie), altitude $560 \mathrm{~m}$, (EK) du 24.V au 19.VI.1996, réc. K. Schiegg.

Autres spécimens: 19, idem, (EK) du 20.VI au 18.VII.1996; 30, idem, (EK) du 23.IV au 21.V.1997; 19 , idem, (EK) du 22.V au 20.VI.1997; réc. K. Schiegg.

Déposés au Musée Zoologique de l'Université Louis Pasteur el de la Ville de Strasbourg.

REMERCIEMENTS. - Nous remencions, très vivement, nos collègues et amis, le Dr J. Boorman, Natural History Museum (Londres) et le Pr. R. Szadziewski, Université de Gdansk (Pologne), pour avoir bien voulu, après examen des données numériques et des figures, donner leur opinion sur ces espèces. Nous tenons également à exprimer toute notre gratitude au Fonds National Suisse de la Recherche Scientifique qui a financé ces recherches $\left(n^{\circ} 31-45911.95\right)$.

\section{AUTEURS CITÉS}

Clastrier J., 1956. - Notes sur les Cératopogonidés. I. Quatre espèces du groupe Forcipomyia d'Algérie et de Tunisie. Archives de l'Institut Pasteur d'Algérie, $34: 496-512$.

Clastrier J., RiouX J.A. \& Descous S., 1961. - Notes sur les Cératopogonidés. XII. Cératopogonidés du Nord-Tchad. Archives de l'Institut Pasteur d'Algérie, 39: 49-98.

DF:IICCOLLE J.-C. \& SCHIEGG K., 1998. - Contribution à l'étude des Cératopogonidés de Suisse. Révision et redescription des espèces paléarctiques du genre Ceratoculicoides Wirth \& Ratanaworabhan, 1971 (Diptera, Nematocera). Bulletin de la Société entomologique de France, 103 (3): 273-286.

— 1999. - Contribution à l'étude des Cératopogonidés de Suisse. II. Description de Brachypogon (s. str.) fagicola $n$. sp. (Diptera, Nematocera). Bulletin de la Société entomologique de France, 104 (1): 31-34.

FUNKE W., 1971. - Food and energy turnover of leaf-eating insects and their influence on primary production. Ecology Studies, 2: 81-93.

KAILA L., 1993. - A new method for collecting quantitative samples of insects associated with decaying wood fungi. Entomologica Fennica, 3: 21-23

KIFFFFR J.-J., 1901. - Synopse des représentants européens du groupe Ceratopogon avec description de quelques espèces nouvelles. Bulletin de la Société d'Histoire naturelle de Moselle (Metz), 9: 143-165.

MACQUART J., 1826. - Insectes Diptères du Nord de la France. Tipulaires. Société des Sciences, de l'Agriculture et des Arts, Lille, 1823-1824: 59-224.

WINNERTZ J., 1852. - Beitrag zur Kenntniss der Gattung Ceratopogon Meigen. Linnaea Entomologica, 6: 1-80. 